\title{
The project of third harmonic medium power W-band gyrotron
}

\author{
$\underline{\text { A.S. Zuev }}{ }^{1}$, A.P. Fokin ${ }^{1}$, M.Y. Glyavin ${ }^{1}$, R.M. Rozental ${ }^{1}$, A.S. Sedov ${ }^{1}$, E.S. Semenov ${ }^{1}$ \\ ${ }^{1}$ IAP RAS, Nizhniy Novgorod, Russia, alan.zuev@yandex.ru
}

One of the most interesting ranges is the W-band due to the presence of a transmission line in this range in the atmosphere, which is used for communication, radiolocation, security and for other applications [14]. These applications require continuous and pulsed $\mathrm{W}$-band sources. One of the powerful devices in this band is the gyrotron [5]. The gyrotron is a vacuum electronics device based on the interaction between an electron beam and microwave fields, which coupling is achieved by the cyclotron resonance condition. High power subterahertz and terahertz radiation gyrotrons require strong external static magnetic field typically provided by a superconducting magnet. Such magnets are quite expensive; moreover they require special operating conditions.

One way to reduce the cost of this device is to develop gyrotrons operating at higher harmonics on the cyclotron frequency, in which the required external magnetic field in the interaction space is significantly less and allows the use of low-cost versions of magnetic systems. At present, there are projects of "warm" (i. e., with liquid or oil cooling) magnets with a cavity diameter sufficient for the gyrotron and the magnetic field level of more than $1 \mathrm{~T}$ [6]. The operation at high harmonics on the cyclotron frequency in gyrotrons encounters the problem of competition of modes, synchronous with lower harmonics of the electron beam. In addition, in order to reach the effective electron-wave interaction for operation at high harmonics, on needs to use resonators with higher quality factor. It leads to an increase in ohmic losses and, consequently, a drop in the output power for the same parameters of the electron beam. In this report, we consider the project of a gyrotron at frequency 95 $\mathrm{GHz}$ of medium power operating at the third harmonic. To solve the problem of mode competition, it is planned to use a relatively unusual method - the addition of an annular diaphragm in the output part of the resonator (fig 1). By introducing the annular diaphragm, it is possible to reduce the diffraction quality factor and, respectively, to increase the starting current of the competing mode.

For the operation of the gyrotron at the third harmonic, mode $\mathrm{TE}_{1,3}$ was chosen. This mode is isolated from modes operating at the fundamental harmonic, and is almost isolated from modes synchronous with the second harmonic. The nearest competitor for it is the counterrunning wave $\mathrm{TE}_{1,2}$ on the second harmonic. The gyrotron was designed for operation at an accelerating voltage $30 \mathrm{kV}$. We used a model with a non-fixed structure of the RF field taking into account the velocity spread of the electron beam. The dependences of the starting currents on the magnetic field for the working and competing modes were calculated and shown in figure 2. The presence of a parasitic mode in the excitation region of the operating mode limits the operating current to $3 \mathrm{~A}$ and ultimately determines the maximum possible output power of the gyrotron. In this case, at the operating current $3 \mathrm{~A}$ the optimum length of resonator is $62 \mathrm{~mm}$ with the output efficiency $5.8 \%$, while the share of ohmic losses is $64 \%$. On the other hand, if one can effectively suppress the parasitic mode, it would be possible to increase the operating current, at which the optimum length of the resonator becomes less. In particular, at operating current $10 \mathrm{~A}$ the maximum efficiency $11.4 \%$ is achieved with the optimum resonator length $49 \mathrm{~mm}$. Figure 3 shows the dependence of the share of ohmic losses and the output efficiency on the resonator length at operating currents $3 \mathrm{~A}$ and $10 \mathrm{~A}$. In addition, the length of the resonator is limited by the characteristic size of the magnetic systems. For further calculations, the resonator length $50 \mathrm{~mm}$ with the output efficiency $4.7 \%$ at current $3 \mathrm{~A}$ and the output efficiency $11.3 \%$ at current $10 \mathrm{~A}$ was chosen. For a longer resonator length it becomes difficult to realize a homogeneous section of the magnetic field in the interaction space.

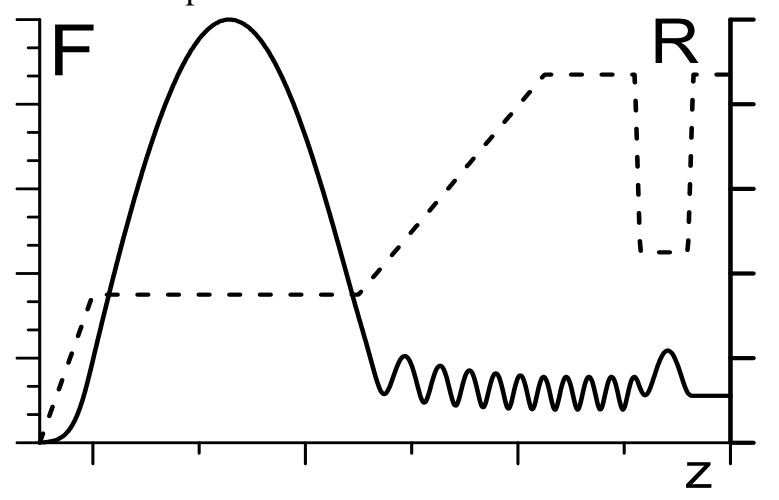

Fig. 1. General view of the cavity profile with an annular diaphragm (dashed line) and longitudinal structure of RF field (solid line).

One of the electrodynamic methods of suppressing the parasitic mode in the gyrotron is the addition of an annular diaphragm in the output part of the resonator. This method of solving the problems of mode competition was proposed earlier, for example, in [7], where the influence of the "external reflector" on the quality factors of the neighboring longitudinal modes was considered. By solution of the cold problem of the longitudinal field structure in the entire volume of the gyrotron electrodynamic system, the parameters of the annular diaphragm for optimal mode selection were chosen. The system with the annular diaphragm drops the diffractive quality factor of the competing $\mathrm{TE}_{1,2}$ mode by more than four times, while the diffraction quality factor of the operating mode remains at the same level. Figure 2 shows that the addition of the annular diaphragm results in a significant increase of the starting current of the parasitic mode $\mathrm{TE}_{1,2}$ in the 
field of excitation of the operating mode. Also the starting current of the $\mathrm{TE}_{1,3}$ mode nearly unchanged. The new electrodynamic structure allows to increase the operating current up to $10 \mathrm{~A}$ and thus to increase the output power by 7 times.

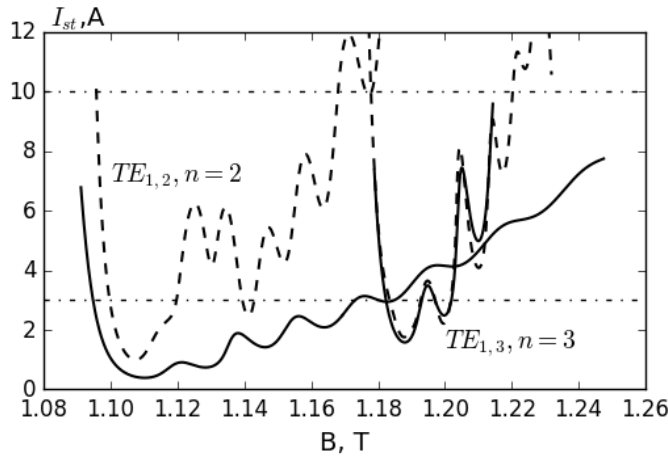

Fig. 2. Dependence of the starting currents on the magnetic field for the operating mode and the main competing mode in a gyrotron at a frequency of $95 \mathrm{GHz}$ with a traditional electrodynamic structure (solid lines) and with diaphragm (dashed lines).

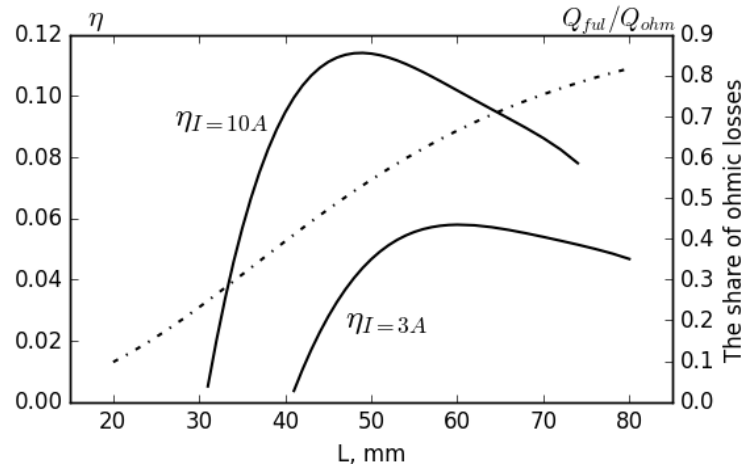

Fig. 3. Dependence of the share of ohmic losses and the output efficiency in the gyrotron on the resonator length at operating currents of $3 \mathrm{~A}$ and $10 \mathrm{~A}$

The estimation of the stability of this method of mode selection for errors in the manufacture of a gyrotron allows us to hope for scaling this method to the region of higher frequencies. Further development of the system consists of optimization of the resonator profile, which will reduce the share of ohmic losses while maintaining high efficiency, and also exclude the possibility of transforming the operating mode into modes with other azimuthal variations. In addition, an experimental verification of this selection method is planned.

The paper is based on the research results, which was conducted in the framework of projects of the Russian Science Foundation No17-79-10422.

\section{References}

1. Bratman, V. L., Litvak, A. G., Suvorov, E. V. Mastering the terahertz domain: sources and applications // PhysicsUspekhi. 2011. V. 54, No. 8, P. 837-870.

2. Kumar, N., Singh, U., Singh, T. P., Sinha, A. K. A Review on the Applications of High Power, High Frequency Microwave Source: Gyrotron // Journal of Fusion Energy. 2011. V. 30, No. 4. P. 257-276.
3. Manheimer, W. M. On the possibility of high power gyrotrons for super range resolution radar and atmospheric sensing // International Journal of Electronics. 1992. V. 72, No. 5-6. P. 1165-1189.

4. Pilossof, M., Einat, M. A $95 \mathrm{GHz}$ mid-power gyrotron for medical applications measurements // Review of Scientific Instruments. 2015. V. 86, No. 1. P. 016113.

5. Nusinovich, G. S., Thumm, M. K. A., Petelin, M. I. The Gyrotron at 50: Historical Overview // Journal of Infrared, Millimeter, and Terahertz Waves. 2014. V. 35, No. 4. P. 325-381.

6. Proyavin, M. D., Glyavin, M. Yu., Manuilov, V. N. Development of high-efficient gyrotron based complex for industrial applications. // EPJ Web Conf. 2017. No. 149, P. 04034.

7. Rozental, P. M., Ginzburg, H. C., Zaitsev, N. I., Ilyakov E.V., Kulagin I.S. Controllable spectrum of an axial-mode gyrotron with external reflections // Tecnical Physics. the Russian Journal of Appl. Phys. 2006. V. 76, No. 1. P. 7881 . 